\title{
Separable quantizations of Stäckel systems
}

\author{
Maciej Błaszak \\ Faculty of Physics, Division of Mathematical Physics, A. Mickiewicz University \\ Umultowska 85, 61-614 Poznań, Poland \\ blaszakm@amu.edu.pl \\ Krzysztof Marciniak \\ Department of Science and Technology \\ Campus Norrköping, Linköping University \\ 601-74 Norrköping, Sweden \\ krzma@itn.liu.se \\ Ziemowit Domański \\ Center for Theoretical Physics of the Polish Academy of Sciences \\ Al. Lotników 32/46, 02-668 Warsaw, Poland \\ domanski@cft.edu.pl
}

October 17, 2018

\begin{abstract}
In this article we prove that many Hamiltonian systems that can not be separably quantized in the classical approach of Robertson and Eisenhardt can be separably quantized if we extend the class of admissible quantizations through a suitable choice of Riemann space adapted to the Poisson geometry of the system. Actually, in this article we prove that for every quadratic in momenta Stäckel system (defined on $2 n$ dimensional Poisson manifold) for which Stäckel matrix consists of monomials in position coordinates there exist infinitely many quantizations - parametrized by $n$ arbitrary functions - that turn this system into a quantum separable Stäckel system.
\end{abstract}

Keywords and phrases: Poisson manifolds, Hamiltonian systems, Darboux coordinates, HamiltonJacobi equation, Schrödinger equation, separability, quantization, Robertson condition, pre-Robertson condition

\section{Introduction}

In classical mechanics the Hamiltonian equations of motion are represented by a system of nonlinear ODE's and are in general not integrable. A famous exception is the class of the so called Liouville integrable systems, i.e. those Hamiltonian systems which possess a sufficient number of global constants of motion in involution. In order to integrate such a system by quadratures it is necessary to find a distinguish orthogonal coordinates, so called separation coordinates. Once we find separation coordinates we can linearize equations of motion according to Hamilton-Jacobi method and then integrate them. Particular important class of separable systems, specially from the physical point of view, is represented in literature by so called Stäckel systems, with Hamiltonian and all constants of motion quadratic in momenta. In the present paper we also restrict ourselves to such class of systems.

This paper deals with admissible quantizations of classical Stäckel systems and investigation of their quantum integrability and quantum separability. Surprisingly, in spite of the fact that there exists an extensive literature on that subject, nevertheless the foundations of the theory have been formulated in the early 1930's by Robertson and Eisenhart (see the next section) and have not been changed until now. In their approach is considered only one particular way of quantization, which we now call natural 
minimal quantization, i.e. the minimal quantization generated by the metric from the kinetic part of the Hamiltonian of the system. One of the results of this classical theory is the so called Robertson condition, the fullfilment of which guarantees the quantum separability of the stationary Schrödinger equation generated by the corresponding quantized Hamiltonian. In consequence, according to RobertsonEisenhart theory, there is only a very limited class of Stäckel systems which are quantum separable.

In this paper we broaden the theory by considering quantizations related to arbitrary metric tensor, not necessarily related with the Hamiltonian of the system. As a consequence of this new approach, we are able to formulate the following conjecture:

\section{For arbitrary Stäckel system with all constants of motion quadratic in momenta there exists a family of quantizations preserving quantum separability.}

In this paper we prove that conjecture for a very large class of Stäckel systems, generated by separation relations of the form (17), where Stäckel matrix consists of monomials in position coordinates. For any Stäckel system from this class we construct a family of metrices for which the minimal quantization leads to quantum separability and commutativity of the quantized constants of motion. We want to stress, however, that we do not deal with spectral theory of the obtained quantum systems, as it requires a separate investigations.

The paper is organized as follows. In Section 2 we briefly summarize the results of Robertson-Eisenhart theory of quantum separability. In Section 3 we present some fundamental facts about classical Stäckel systems. Section 4 contains presentation of some results derived from our general theory of quantization of Hamiltonian systems on phase space; especially we demonstrate how to obtain the minimal quantization (44) from our general theory. In Section 5 we relate quantizations of the same Hamiltonian in different metrics $g$ and $\bar{g}$ (or in different Hilbert spaces $L^{2}\left(Q, \omega_{g}\right)$ and $L^{2}\left(Q, \omega_{\bar{g}}\right)$ ). Essentially, this construction explains the origin of the quantum correction terms in the classical Hamiltonians introduced in [1] and in 2]. Section 6 is devoted to the issue of separable quantizations of Stäckel systems. We construct a family of metric tensors which fulfill the so called generalized Robertson condition introduced in our previous paper [3]. Using this condition we prove (Theorem 8) that there exists an infinite family, parametrized by $n$ arbitrary functions of one variable, of separable quantizations of a given Stäckel system from our considered class. Finally, in Section 7 we address the issue of quantum integrability of Stäckel systems. This section generalizes in an essential way the results from [4]. We present the construction of commuting self-adjoint operators in arbitrary Hilbert spaces $L^{2}\left(Q, \omega_{g}\right)$, once we have a quantum separable Stäckel system. It also contains two illustrative examples. An invariant form of Theorem 6 is proved in Appendix.

\section{Preliminaries - legacy of Robertson and Eisenhardt}

This paper addresses the issue of separable and integrable quantizations of commuting sets of quadratic in momenta Hamiltonians of the form

$$
H(x, p)=\frac{1}{2} A^{i j}(x) p_{i} p_{j}+V(x)
$$

(throughout the whole article we apply - unless explicitly stated otherwise - the Einstein summation convention) defined on a cotangent bundle to some $n$-dimensional Riemannian manifold $Q$ equipped with metric tensor $g$. The variables $x=\left(x_{1}, \ldots, x_{n}\right)$ are coordinates on $Q$ and $p_{i}$ conjugate momenta (fiber coordinates in $\left.T^{*} Q\right)$ while $A^{i j}(x)$ are components of a symmetric $(2,0)$-tensor $A$ on $Q$. Note that we do not assume here any relation between the tensor $A$ and the metric tensor $g$. The real function $V(x)$ is called the potential of the Hamiltonian (11). Two important partial differential equations can be associated with the Hamiltonian (1): the Hamilton-Jacobi equation

$$
H\left(x_{1}, \ldots, x_{n}, \frac{\partial W}{\partial x_{1}}, \ldots, \frac{\partial W}{x_{n}}\right)=a
$$

for the generating function $W(x, a)$ for a canonical transformation linearizing the flow of Hamilton equations

$$
x_{i, t}=\frac{\partial H}{\partial p_{i}}, \quad p_{i, t}=-\frac{\partial H}{\partial x_{i}}, \quad i=1, \ldots, n
$$


(here and in what follows the comma denotes the differentiation with respect to a variable) associated with (11), and the stationary Schrödinger equation

$$
\hat{H} \Psi(x)=E \Psi(x)
$$

where

$$
\hat{H}=-\frac{\hbar^{2}}{2} \nabla_{i} A^{i j} \nabla_{j}+V(x)
$$

is the Hamilton operator (quantum Hamiltonian) acting on the Hilbert space $L^{2}\left(Q,|\operatorname{det} g|^{1 / 2} d x\right)$ of square integrable (in the measure $\omega_{g}=|\operatorname{det} g|^{1 / 2} d x$ ) complex functions on $Q$. The operators $\nabla_{i}$ are operators of Levi-Civita connection associated with the metric $g$ and $\hbar$ is the Planck constant. One says then that the Hamilton operator (44) is the quantization of the Hamiltonian (11) in the metric $g$. Note that the above quantization procedure is so far defined ad hoc, arbitrarily.

An important issue related with equations (2) and (3) is the problem of their separability. We say that the Hamilton-Jacobi equation (2) is additively separable if it admits a solution

$$
W(x, a)=\sum_{i=1}^{n} W_{i}\left(x_{i}, a\right)
$$

depending in a suitable manner on $n$ additional parameters $a=\left(a_{1}, \ldots, a_{n}\right)$ (the solution (5) is often called a complete integral of (2)). Similarly, we say that the Schrödinger equation (3) is multiplicatively separable if it admits a solution

$$
\Psi(x, a)=\prod_{i=1}^{n} \psi_{i}\left(x_{i}, a\right)
$$

depending in a suitable way on $2 n$ additional parameters $a=\left(a_{1}, \ldots, a_{2 n}\right)$. P. Stäckel showed in [5] the necessary and sufficient conditions for separability of (1) in orthogonal (with respect to $A$ ) coordinates (meaning that $A$ has to be diagonal in the variables $x$ ). Assume thus that $A$ plays the role of the contravariant metric (i.e. that $A=G$, where $G=g^{-1}$ ) and that the metric $G$ is diagonal in coordinates $x$. Robertson [6] proved that in this case if the Hamilton-Jacobi equation (2) separates in the variables $x$ then the Schrödinger equation (3) also separates provided that an additional condition, called today Robertson condition, is satisfied. Eisenhart in [7] proved that Robertson condition is satisfied if and only if the Ricci tensor $R_{i j}$ of the metric $g$ is diagonal. We stress again that in these works $A=G$ and in this particular case the Hamilton operator (4) takes the form

$$
\hat{H}=-\frac{\hbar^{2}}{2} G^{i j} \nabla_{i} \nabla_{j}+V(x)
$$

Robertson actually claimed in his theorem that the separability of Schrödinger equation also implies separability of Hamilton-Jacobi equation; this statement is not correct if we use the definition of separability used by Robertson. Benenti et al in [8] completed the works of Robertson and Eisenhart by introducing an appropriate definition of separability of Schrödinger equation, involving $2 n$ parameters $a_{i}$ as in (6) (Robertson had no parameters in his definition of separability, a drawback not observed by Eisenhart). Assuming the definition of Benenti et al the theorem of Robertson becomes:

Theorem 1 Assume that $A=G$ and that $G$ is diagonal in the variables $x_{i}$. The Schrödinger equation (3) admits a separable solution (6) if and only if the Hamilton-Jacobi equation (2) admits a separable solution (5) and moreover if the Robertson condition

$$
R_{i j}=0 \text { for all } i \neq j
$$

is satisfied.

One can show that in orthogonal coordinates

$$
R_{i j}=\frac{3}{2} \partial_{i} \Gamma_{j}, \quad i \neq j
$$


where $\Gamma_{i}$ are metrically contracted Christoffel symbols of $g$ defined by

$$
\Gamma_{i}=g_{i l} G^{j k} \Gamma_{j k}^{l}, \quad i=1, \ldots, n
$$

Thus, in orthogonal coordinates the Robertson condition becomes

$$
\partial_{i} \Gamma_{j}=0 \text { for } j \neq i
$$

In papers [6] and [7] the authors considered a quantization procedure for only one Hamiltonian and assumed that the underlying metric of the configuration space is defined by the tensor $A$ in the Hamiltonian, i.e. they assumed that $A=G$. Suppose now that we have $n(n=\operatorname{dim} Q)$ Poisson-commuting (so they constitute an integrable system in the sense of Liouville) Hamiltonians each of the form (10):

$$
H_{r}=\frac{1}{2} A_{r}^{i j} p_{i} p_{j}+V_{r}(x), \quad r=1, \ldots, n .
$$

A natural question one can pose is whether the corresponding quantum Hamiltonians $\hat{H}_{r}$ (acting in the Hilbert space $L^{2}\left(Q, \omega_{g}\right)$ defined by the metric $\left.G=A_{1}\right)$ will constitute a quantum integrable systems i.e. whether they will commute. In [4] the authors proved that this happens if and only if the so called pre-Robertson condition

$$
\partial_{i} R_{i j}-\Gamma_{i} R_{i j}=0, \quad i \neq j
$$

is satisfied. Due to (8), this condition in orthogonal coordinates reads

$$
\partial_{i}^{2} \Gamma_{j}-\Gamma_{i} \partial_{i} \Gamma_{j}=0, \quad i \neq j .
$$

Remark 2 The Robertson condition (7) or (10) implies the pre-Robertson condition (12) or (13) so quantum separability implies the quantum integrability, as it is in the classical case.

The above theory describes the quantization of a Hamiltonian, or a set of Hamiltonians, of the form (1) in the case when one of the tensors $A_{r}$ plays the role of the metric. However, Hamiltonians are functions on a phase space with no obvious metric given. In this paper we will therefore develop the theory of quantization of Hamiltonians of type (10) in Hilbert spaces $L^{2}\left(Q, \omega_{g}\right)$ defined by the metric not related to these Hamiltonians. Let us thus pose the following question: given a separable Hamiltonian system consisting of $n$ Hamiltonians of the form (11), how to find metric tensor(s) in which an appropriate quantization procedure turns this system into a separable and integrable quantum system? We will answer this question in the spirit of papers 9 -11] where we have developed a general theory of quantizing Hamiltonian systems directly on the phase space; the quantization in this approach is given by an appropriate deformation of Poisson algebra of classical observables (real functions) on the phase space $M$ to a quantum algebra. Various deformations of this algebra are related to each other by an automorphism $S$. However, in order to make this article as compact as possible, we will almost completely omit this general setting but use its results in the position representation, that is, we will work directly in Hilbert spaces $L^{2}\left(Q, \omega_{g}\right)$ of the functions defined on the base manifold $Q$.

\section{Classical Stäckel systems in separation coordinates and adapted Riemannian geometry}

Consider a $2 n$-dimensional connected Poisson manifold $(M, \mathcal{P})$, where $\mathcal{P}$ is a non-degenerated Poisson tensor. An integrable system is a set of $n$ real valued functions $H_{i}$ on $M$ in involution with respect to a Poisson bracket:

$$
\left\{H_{i}, H_{j}\right\}:=\mathcal{P}\left(d H_{i}, d H_{j}\right)=0, \quad i, j=1, \ldots, n .
$$

The functions $H_{i}$ generate $n$ pairwise commuting Hamiltonian equations

$$
u_{, t_{i}}=\mathcal{P} d H_{i}, \quad i=1, \ldots, n, \quad u \in M .
$$

i.e. an integrable system. Let us fix a set $(x, p)=\left(x_{1}, \ldots x_{n}, p_{1}, \ldots p_{n}\right)$ of Darboux (canonical) coordinates on $M$ (so that $\left\{x_{i}, x_{j}\right\}=\left\{p_{i}, p_{j}\right\}=0,\left\{x_{i}, p_{j}\right\}=\delta_{i j}$ ). One of the methods of solving the equations 
(14) is to find a solution $W(x, a)$ to the system of Hamilton-Jacobi equations (2) corresponding to the Hamiltonians $H_{i}$

$$
H_{i}\left(x_{1}, \ldots, x_{n}, \frac{\partial W}{\partial x_{1}}, \ldots, \frac{\partial W}{x_{n}}\right)=a_{i}, \quad i=1, \ldots, n .
$$

The solution $W(x, a)$ is then a generating function for a canonical transformation $(x, p) \mapsto(b, a)$ to a new set of coordinates on $M$ (with $a_{i}=H_{i}$ ) in which the equations (14) attain the form

$$
b_{i, t_{j}}=\delta_{i j}, \quad a_{i, t_{j}}=0
$$

so that all the flows in (14) linearize in coordinates $(b, a)$. In most cases the system of PDE's (15) is a highly nonlinear system that is very difficult to solve. However, as we mentioned in introduction, a very appealing situation occurs if we can find Darboux coordinates $(\lambda, \mu)=\left(\lambda_{1}, \ldots \lambda_{n}, \mu_{1}, \ldots \mu_{n}\right)$ in which there exists a complete integral for all the Hamilton-Jacobi equations (15) of the form

$$
W(\lambda, a)=\sum_{i=1}^{n} W_{i}\left(\lambda_{i}, a\right)
$$

(see (5)) where each function $W_{i}$ depend only on one canonical coordinate $\lambda_{i}$ and in a nontrivial way on all parameters $a=\left(a_{1}, \ldots, a_{n}\right)$. In such a case the systems of PDE's (15) split into $n$ uncoupled ODE's for the functions $W_{i}$, which makes it possible to solve them by quadratures. The coordinates $(\lambda, \mu)$ are then called separation coordinates of the system (14).

The most convenient way to obtain separable systems is to define them directly in separation coordinates. It is done with the help of the so called separation relations [12], i.e. $n$ algebraic relations of the form

$$
\varphi_{i}\left(\lambda_{i}, \mu_{i}, a_{1}, \ldots, a_{n}\right)=0, \quad i=1, \ldots, n
$$

each depending on one pair of canonical coordinates and on parameters $a_{i}$. If there exists an open dense set $\Omega \subset M$ on which the relations (16) can be solved with respect to the coefficients $a_{i}$ yielding

$$
a_{i}=H_{i}(\lambda, \mu), \quad i=1, \ldots, n
$$

then it is easy to show that the functions $H_{i}$ Poisson commute (i.e. constitute a Liouville integrable system as defined above) and moreover that the coordinates $(\lambda, \mu)$ are separation coordinates for the Hamiltonians $H_{i}$.

One of the most important classes of separable systems are the so called Stäckel systems, introduced by P. Stäckel in [5] and thoroughly studied in literature (see for example [13 15]). They are generated by separation relations linear in Hamiltonians $H_{i}$ and quadratic in canonical momenta $\mu_{i}$. In our paper we restrict ourselves to a — still very general — class of Stäckel systems defined by the following separation relations

$$
H_{1} \lambda_{i}^{\gamma_{1}}+H_{2} \lambda_{i}^{\gamma_{2}}+\cdots+H_{n} \lambda_{i}^{\gamma_{n}}=\frac{1}{2} f_{i}\left(\lambda_{i}\right) \mu_{i}^{2}+\sigma_{i}\left(\lambda_{i}\right), \quad i=1, \ldots, n,
$$

where $\gamma_{i}$ are natural numbers such that $\gamma_{1}>\gamma_{2}>\cdots>\gamma_{n}=0$ (the last choice is for our convenience only) have no common divisor, and where $f_{i}, \sigma_{i}$ are some rational functions of one argument. The separation relations (17) can be written in a matrix form as

$$
S_{\gamma} H=U
$$

where $H=\left(H_{1}, \ldots, H_{n}\right)^{T}$ and $U=\left(\frac{1}{2} f_{1}\left(\lambda_{1}\right) \mu_{1}^{2}+\sigma_{1}\left(\lambda_{1}\right), \ldots, \frac{1}{2} f_{n}\left(\lambda_{n}\right) \mu_{n}^{2}+\sigma_{n}\left(\lambda_{n}\right)\right)^{T}$ is a Stäckel vector and where the matrix $S_{\gamma}$ given by

$$
S_{\gamma}=\left(\begin{array}{cccc}
\lambda_{1}^{\gamma_{1}} & \lambda_{1}^{\gamma_{2}} & \ldots & 1 \\
\vdots & \vdots & & 1 \\
\lambda_{n}^{\gamma_{1}} & \lambda_{n}^{\gamma_{2}} & \ldots & 1
\end{array}\right)
$$

is a particular Stäckel matrix with functions being monomials parametrized by the natural numbers $\gamma_{i}$. We can now take as the set $\Omega$ what remains of $M$ after removing the set of points where det $S_{\gamma}=0$ as well as all the poles of $f_{i}$ and $\sigma_{i}$. Solving the relations (18) on $\Omega$ we obtain the Stäckel Hamiltonians 


$$
H_{r}=\frac{1}{2} \mu^{T} A_{r} \mu+V_{r}(\lambda), \quad r=1, \ldots, n
$$

with

$$
A_{r}=\operatorname{diag}\left(\left(S_{\gamma}^{-1}\right)_{r 1} f_{1}\left(\lambda_{1}\right), \ldots,\left(S_{\gamma}^{-1}\right)_{r n} f_{n}\left(\lambda_{n}\right)\right)
$$

being diagonal matrices with entries that are functions of $\lambda$-variables only and with the potentials of the form

$$
V_{r}=\sum_{i}\left(S_{\gamma}^{-1}\right)_{r i} \sigma_{i}\left(\lambda_{i}\right) \quad r=1, \ldots, n
$$

The systems of the above class, albeit not general Stäckel systems, still encompass majority of the Stäckel systems considered in literature.

Let us now introduce some Riemannian geometry into our considerations. The specifications below will be motivated by the fact that our quantization procedure will be performed in appropriate (pseudo-)Riemannian spaces. Thus, from now on we will suppose that our manifold $M$ is a cotangent bundle to some pseudo-Riemannian manifold i.e. $M=T^{*} Q$ with $Q$ equipped with some metric tensor $g$. We will also make three additional assumptions:

1. The manifold $(Q, g)$ and the Poisson structure are adapted to each other in the sense that the first $n$ Darboux coordinates $\lambda_{i}$ are coordinates on $Q$ while the remaining Darboux coordinates $\mu_{i}$ are fiber coordinates.

2. Coordinates $\lambda_{i}$ are orthogonal coordinates for the metric $g$ i.e. $g$ and $G=g^{-1}$ are diagonal (but not necessarily flat) in $\lambda_{i}$.

3. The base manifold $Q$ is almost covered by a single, open and dense in $M$, chart with coordinates $\left(\lambda_{1}, \ldots, \lambda_{n}\right)$.

The matrices $A_{r}$ in (19) can now be interpreted as $(2,0)$-tensors on $Q$ that can be written as

$$
A_{r}=T_{r} G, \quad r=1, \ldots, n
$$

where $T_{r}$ are $(1,1)$-tensors on $Q$. Further, in a very special case when $G=A_{1}$ the tensors $T_{r}$ are Killing tensors for the metric $G$. We will denote them as $K_{r}$, so that

$$
A_{r}=K_{r} A_{1}, \quad r=1, \ldots, n
$$

A particular subclass of Stäckel systems (17) is then given by choosing $\gamma_{i}=n-i$. Such systems are called Stäckel system of Benenti type (or simply Benenti systems) and are thus generated by the separation relations of the form

$$
H_{1} \lambda_{i}^{n-1}+H_{2} \lambda_{i}^{n-2}+\cdots+H_{n}=\frac{1}{2} f_{i}\left(\lambda_{i}\right) \mu_{i}^{2}+\sigma_{i}\left(\lambda_{i}\right), \quad i=1, \ldots, n
$$

It can be shown that in the Benenti case the metric tensor $G=A_{1}$ has the form

$$
A_{1}=\operatorname{diag}\left(\frac{f_{1}\left(\lambda_{1}\right)}{\Delta_{1}}, \ldots, \frac{f_{n}\left(\lambda_{n}\right)}{\Delta_{n}}\right), \quad \Delta_{i}=\prod_{j \neq i}\left(\lambda_{i}-\lambda_{j}\right)
$$

while the Killing tensors $K_{r}$ are of the form

$$
K_{r}=-\operatorname{diag}\left(\frac{\partial \rho_{r}}{\partial \lambda_{1}}, \cdots, \frac{\partial \rho_{r}}{\partial \lambda_{n}}\right), \quad r=1, \ldots, n
$$

with $\rho_{i}=\rho_{i}(\lambda)$ being signed symmetric polynomials (Viète polynomials) in the variables $\lambda_{1}, \ldots, \lambda_{n}$ :

$$
\rho_{i}(\lambda)=(-1)^{i} \sum_{1 \leq s_{1}<s_{2}<\ldots<s_{i} \leq n} \lambda_{s_{1}} \ldots \lambda_{s_{i}}, \quad i=1, \ldots, n
$$


Let us now go back to an arbitrary Stäckel system of the form (17) defined by the choice of the constants $\gamma_{1}>\gamma_{2}>\cdots>\gamma_{n}=0$ and the choice of functions $f_{i}, \sigma_{i}$. Then the tensors $A_{r}$ for this system can be written as $[13]$

$$
A_{r}=\frac{1}{\varphi} \chi_{r} G_{B, f}, \quad r=1, \ldots, n
$$

where $G_{B, f}$ is the corresponding Benenti metric given by (22)

$$
G_{B, f}=\operatorname{diag}\left(\frac{f_{1}\left(\lambda_{1}\right)}{\Delta_{1}}, \ldots, \frac{f_{n}\left(\lambda_{n}\right)}{\Delta_{n}}\right)
$$

where $\chi_{r}$ are some polynomial functions of the Killing tensors $K_{r}$ in (23) and where

$$
\varphi=\operatorname{det}\left(\begin{array}{ccc}
\rho_{n_{1}-1} & \cdots & \rho_{n_{1}-k} \\
\vdots & \ddots & \vdots \\
\rho_{n_{k}-1} & \cdots & \rho_{n_{k}-k}
\end{array}\right)
$$

(where we adapt the notation $\rho_{0}=1$ and $\rho_{i}=0$ for $i<0$ or $i>n$ ) while the constants $n_{i}$ are those for which the corresponding monomials $\lambda^{n+k-n_{i}}$ are missing in the left hand side of (17) (they are "holes" in the sequence $\left\{\gamma_{1}=n+k-1, \gamma_{2}, \cdots, \gamma_{n}=0\right\}$ numbered from the left; $k$ is determined from the equation $\gamma_{1}=n+k-1$ ). Note that if such "holes" are absent (as in Benenti case) then $\varphi=1$. For example, if the left hand side of the Stäckel system is $H_{1} \lambda^{4}+H_{2} \lambda+H_{3}$, then $n=3, k=2, n_{1}=2, n_{2}=3$ and the function (27) becomes:

$$
\varphi=\operatorname{det}\left(\begin{array}{cc}
\rho_{1} & \rho_{0} \\
\rho_{2} & \rho_{1}
\end{array}\right)=\lambda_{1}^{2}+\lambda_{1} \lambda_{2}+\lambda_{1} \lambda_{3}+\lambda_{2}^{2}+\lambda_{2} \lambda_{3}+\lambda_{3}^{2}
$$

We finish this chapter with an important remark.

Remark 3 If $f_{i}=f$ and if $f$ is a polynomial of order $\leq n$ then the metric $G_{B, f}$ in (26) is flat.

\section{Admissible quantizations of quadratic in momenta Hamilto- nians on pseudo-Riemannian spaces}

A usual way of quantization of a given Hamiltonian system living on a phase space $M=\mathbf{R}^{2 n}$ is by replacing the observables of the system (i.e. real functions on the phase space of the system, written as functions of positions $x_{i}$ and momenta $p_{i}$ ) by self-adjoint operators acting on the Hilbert space $\mathcal{H}=$ $L^{2}\left(\mathbf{R}^{n}\right)$ of square integrable complex functions on $\mathbf{R}^{n}$. This is done by replacing $x_{i}$ and $p_{i}$ in the observables by the non-commuting operators $\hat{x}_{j}=x_{j}$ and $\hat{p}_{j}=-i \hbar \partial / \partial x_{j}$ acting on $L^{2}\left(\mathbf{R}^{n}\right)$. In this procedure we have to agree on a certain order of non-commuting operators $\hat{x}_{j}$ and $\hat{p}_{j}$ in the obtained operator. One usually applies the Weyl ordering that guarantees that the obtained operators will be self-adjoint.

Suppose now that we want to quantize in a coordinate-free way a Hamiltonian system given on a phase space $M=T^{*} Q$ that is the cotangent bundle to a pseudo-Riemannian manifold equipped with a metric tensor $g$. In a series of papers [9 11] we have developed a consistent theory of quantizing a Hamiltonian system directly on the phase space $M$ through a very general procedure of quantization. Here we briefly sketch some parts of this construction that are important for our further considerations; we perform the construction in the so called position representation.

Let us thus choose a canonical (Darboux) coordinate system $\left(x_{j}, p_{j}\right)$ on $M$ satisfying assumptions 1-3 from the previous section. Thus, $x_{i}$ are some coordinates on $Q$ and $p_{j}$ are the corresponding conjugate momenta. Let us also (following [10], [16], and [17]) introduce the operators

$$
\hat{x}_{j}=x_{j}, \quad \hat{p}_{j}=-i \hbar\left(\frac{\partial}{\partial x_{j}}+\frac{1}{2} \Gamma_{j k}^{k}\right)
$$


acting on the Hilbert space $\mathcal{H}=L^{2}\left(Q, \omega_{g}\right)$ of functions on the base manifold $Q$ (configuration space) where $\omega_{g}=|\operatorname{det} g|^{1 / 2} d x$ is a volume form defined by the metric $g$ and where $\Gamma_{j k}^{k}$ are contracted Christoffel symbols of the metric $g$. The operators (28) are self-adjoint in $\mathcal{H}$ and moreover are canonical quantum operators as $\left[\hat{x}_{j}, \hat{p}_{k}\right]=i \hbar \delta_{j k}$.

Now, a given observable $H=H(x, p)$ can be quantized in many different ways by applying different orderings to the operators $\hat{x}, \hat{p}$ in $H(\hat{x}, \hat{p})$. This can be systematically done using a two-parameter family of automorphisms $S$, introduced in [11], acting on the space of functions on $M$. Any automorphism $S$ from this family relates a given quantization with a Moyal quantization corresponding to our chosen Darboux coordinates $(x, p)$.

Our two-parameter family of automorphisms $S$ is up to $\hbar^{2}$-terms given by

$$
\begin{aligned}
S= & 1+S_{2} \hbar^{2}+o\left(\hbar^{4}\right) \\
= & 1+\frac{\hbar^{2}}{4 !}\left[3\left(\Gamma_{l j}^{i} \Gamma_{i k}^{l}+a R_{j k}\right) \partial_{p_{j}} \partial_{p_{k}}+3 \Gamma_{j k}^{i} \partial_{x_{i}} \partial_{p_{j}} \partial_{p_{k}}+\left(2 \Gamma_{n l}^{i} \Gamma_{j k}^{n}-\Gamma_{j k, l}^{i}\right) p_{i} \partial_{p_{j}} \partial_{p_{k}} \partial_{p_{l}}\right. \\
& \left.-3 b \partial_{p_{j}}\left(\partial_{x_{j}}+\Gamma_{j l}^{i} p_{i} \partial_{p_{l}}\right) \partial_{p_{k}}\left(\partial_{x_{k}}+\Gamma_{k n}^{r} p_{r} \partial_{p_{n}}\right)\right]+o\left(\hbar^{4}\right),
\end{aligned}
$$

( $a$ and $b$ are real parameters and $\Gamma_{j k, l}^{i}=\partial_{x_{l}} \Gamma_{j k}^{i}$ ) with the inverse given formally by

$$
S^{-1}=1-S_{2} \hbar^{2}+o\left(\hbar^{4}\right)
$$

Remark 4 The terms o $\left(\hbar^{4}\right)$ in (67) are at least of the fourth order in $\partial_{p_{j}}$ so the formulas (67)-(30) are enough to calculate the action of $S$ respectively $S^{-1}$ on Hamiltonians that are up to third order in momenta.

We can now introduce the following quantization procedure of a given observable $H(x, p)$ :

1. Deformation of $H(x, p)$ to a new function $H^{\prime}(x, p)=S^{-1} H$ by an automorphism $S$ from our family (67)

2. Replacing $x_{j}$ and $p_{j}$ in $H^{\prime}(x, p)$ by the operators (28), which yields the operator $H^{\prime}(\hat{x}, \hat{p})$

3. Weyl ordering of the obtained operator.

In short, the $S$-quantization of $H(x, p)$ in the metric $g$ is the operator

$$
\hat{H}=\left(S^{-1} H\right)_{W}(\hat{x}, \hat{p})
$$

(where $W$ denotes the Weyl ordering) with operators $\hat{x}, \hat{p}$ given by (28) and with a chosen automorphism $S$ from our two-parameter family. It can be shown that this procedure applied to any classical (real) observable on $M$ yields a self-adjoint operator on $\mathcal{H}=L^{2}\left(Q, \omega_{g}\right)$.

Remark 5 The presented procedure is invariant under the canonical change of coordinates in the sense that if we start from another canonical set of coordinates satisfying assumptions 1-3 from the previous section we obtain the quantum operator that is unitarily equivalent to $\hat{H}$.

Applying the above quantization procedure with the automorphism $S$ as in (67) to a quadratic in momenta Hamiltonian

$$
H=\frac{1}{2} p^{T} A p+V(x)
$$

yields the two-parameter family of operators (quantum Hamiltonians) on $\mathcal{H}[11]$ :

$$
\begin{aligned}
\hat{H} & =-\frac{\hbar^{2}}{2}\left(\nabla_{i} A^{i j} \nabla_{j}+\frac{1}{4}(1-b) A_{; i j}^{i j}-\frac{1}{4}(1-a) A^{i j} R_{i j}\right)+V(x) \\
& =-\frac{\hbar^{2}}{2} \nabla_{i} A^{i j} \nabla_{j}+\hbar^{2} V_{\text {quant }}(x)+V(x)
\end{aligned}
$$


where $\nabla_{i}$ is the operator of the covariant derivative of the Levi-Civita connection defined by $g, R_{i j}$ is the Ricci tensor of $g$ and where the semicolon ; denotes the covariant derivative. The term $V_{\text {quant }}(x)$ can be considered as a "quantum correction" to the potential $V$ that comes from the quantization process. All considered in literature quantizations of quadratic in momenta Hamiltonians can be obtained by choosing appropriate values of $a$ and $b$ in (33). In the special case when $A=G$ the formula (33) reduces to

$$
\hat{H}=-\frac{\hbar^{2}}{2}\left(G^{i j} \nabla_{i} \nabla_{j}-\frac{1}{4}(1-a) R\right)+V(x)
$$

where $R$ is the Ricci scalar. In the flat case (so that $R_{i j}=0$ ) and with $b=0$ we obtain the Weyl quantization written in a covariant form, and (33) and its specification (34) attain the form

$$
\hat{H}=-\frac{\hbar^{2}}{2}\left(\nabla_{i} A^{i j} \nabla_{j}+\frac{1}{4} A_{; i j}^{i j}\right)+V(x)
$$

and

$$
\hat{H}=-\frac{\hbar^{2}}{2} G^{i j} \nabla_{i} \nabla_{j}+V(x)
$$

respectively.

As we see, in the general quantization scheme there appear the quantum correction term $V_{\text {quant }}(x)$ to the potential $V$. This quantum potential is in general non-separable [18], so from the point of quantum separability the optimal choice of quantization is given by $a=b=1$, which yields

$$
\hat{H}=-\frac{\hbar^{2}}{2} \nabla_{i} A^{i j} \nabla_{j}+V(x)
$$

This quantizations is called a minimal quantization induced by the metric tensor $g$ and $V_{\text {quant }}(x)=0$ in that case. It is exactly the a priori quantization considered by Eisenhardt, Robertson, Benenti and many others and described in the preliminary part above. Our theory clearly explains its origin and shows that this is but one of infinitely many possibilities of quantizing the Hamiltonian (11).

\section{Minimal quantization in different metric spaces}

Our goal now is to relate two minimal quantizations induced by different metric tensors. We will need this in order to be able to write systems of commuting operators in various Hilbert spaces with measures induced by different metrics.

Consider thus two different metric tensors $g$ and $\bar{g}$. As usual, we will denote their contravariant forms by $G$ and $\bar{G}$, respectively. Each of these metrics induces a minimal quantization (described in Section 4 ) by morphisms $S$ and $\bar{S}$, respectively, where (cf. (67) with $a=b=1$ )

$$
\begin{aligned}
S= & 1+\frac{\hbar^{2}}{4 !}\left[3\left(\Gamma_{l j}^{i} \Gamma_{i k}^{l}+R_{j k}\right) \partial_{p_{j}} \partial_{p_{k}}+3 \Gamma_{j k}^{i} \partial_{x_{i}} \partial_{p_{j}} \partial_{p_{k}}+\left(2 \Gamma_{n l}^{i} \Gamma_{j k}^{n}-\Gamma_{j k, l}^{i}\right) p_{i} \partial_{p_{j}} \partial_{p_{k}} \partial_{p_{l}}\right. \\
& \left.-3 \partial_{p_{j}}\left(\partial_{x_{j}}+\Gamma_{j l}^{i} p_{i} \partial_{p_{l}}\right) \partial_{p_{k}}\left(\partial_{x_{k}}+\Gamma_{k n}^{r} p_{r} \partial_{p_{n}}\right)+o\left(\hbar^{4}\right)\right]
\end{aligned}
$$

and where $\bar{S}$ is given by an analogous expression with $\Gamma_{j k}^{i}$ replaced by Christoffel symbols $\bar{\Gamma}_{j k}^{i}$ of the Levi-Civita connection induced by $\bar{g}$. For a (classical) observable of the form

$$
H(x, p)=\frac{1}{2} A^{i j}(x) p_{i} p_{j}+V(x)
$$

by (31), its minimal quantization with respect to $g$ is given by

$$
\hat{H}=\left(S^{-1} H\right)_{W}(\hat{x}, \hat{p})=-\frac{\hbar^{2}}{2} \nabla_{i} A^{i j} \nabla_{j}+V(x)
$$

and acts in $L^{2}\left(Q, \omega_{g}\right)$, while its quantization with respect to $\bar{g}$ is given by a similar expression involving $\bar{\nabla}_{i}$ (that is the covariant differentiation with respect to $\bar{g}$ ) and the operators $\hat{\bar{x}}_{j}=x_{j}$ and 
$\left.\hat{\bar{p}}_{j}=-i \hbar\left(\partial_{j}+\frac{1}{2} \bar{\Gamma}_{j k}^{k}\right)\right)$. These are in general two different operators, acting in two different Hilbert spaces: $L^{2}\left(Q, \omega_{g}\right)$ and $L^{2}\left(Q, \omega_{\bar{g}}\right)$, respectively. The Hilbert spaces $L^{2}\left(Q, \omega_{g}\right)$ and $L^{2}\left(Q, \omega_{\bar{g}}\right)$ are however isometric, with the isometry $L^{2}\left(Q, \omega_{g}\right) \rightarrow L^{2}\left(Q, \omega_{\bar{g}}\right)$ given by

$$
\bar{\Psi}=U \Psi=\frac{|\operatorname{det} g|^{1 / 4}}{|\operatorname{det} \bar{g}|^{1 / 4}} \Psi
$$

where $\Psi \in L^{2}\left(Q, \omega_{g}\right)$ and $\bar{\Psi} \in L^{2}\left(Q, \omega_{\bar{g}}\right)$. The isometry (39) induces a similarity map between operators in both spaces: it maps an operator $\hat{F}$ acting in $L^{2}\left(Q, \omega_{g}\right)$ to the operator

$$
\hat{\bar{F}}=U \hat{F} U^{-1}
$$

acting in $L^{2}\left(Q, \omega_{\bar{g}}\right)$.

Theorem 6 Suppose that the operator $\hat{H}$ in the Hilbert space $L^{2}\left(Q, \omega_{g}\right)$ is given by (38). Then the operator $U \hat{H} U^{-1}$, acting in the Hilbert space $L^{2}\left(Q, \omega_{\bar{g}}\right)$, has the form

$$
U \hat{H} U^{-1}=-\frac{\hbar^{2}}{2} \bar{\nabla}_{i} A^{i j} \bar{\nabla}_{j}+V(x)+\hbar^{2} W(x)
$$

with $W(x)$ given by

$$
W(x)=\frac{1}{8}\left[A^{i j}\left(\Gamma_{i k}^{k} \Gamma_{j s}^{s}-\bar{\Gamma}_{i k}^{k} \bar{\Gamma}_{j s}^{s}\right)+2\left(A^{i j}\left(\Gamma_{j k}^{k}-\bar{\Gamma}_{j k}^{k}\right)\right)_{, i}\right]
$$

where the subscript ${ }_{i}$ denotes differentiation with respect to $x_{i}$.

We will call the term $W(x)$ the quantum correction term as it describes what happens to the operator (38) transformed from $L^{2}\left(Q, \omega_{g}\right)$ to $L^{2}\left(Q, \omega_{\bar{g}}\right)$.

Proof. One can prove this theorem by direct calculations of $U \hat{H} U^{-1}$. Of course

$$
U \hat{H} U^{-1}=U\left(-\frac{\hbar^{2}}{2} \nabla_{i} A^{i j} \nabla_{j}+V(x)\right) U^{-1}=-\frac{\hbar^{2}}{2} U \nabla_{i} A^{i j} \nabla_{j} U^{-1}+V(x)
$$

By using the fact

$$
\frac{\partial U}{\partial x^{i}}=\frac{1}{2} U\left(\Gamma_{i k}^{k}-\bar{\Gamma}_{i k}^{k}\right)
$$

after some calculations we arrive at (41)-(42). Alternatively, the similarity map (40) can be calculated using the automorphism $\bar{S} S^{-1}$. From our general theory [9]-[11] it follows that quantizing the observable $H$ with respect to $g$ yields an operator that is mapped through (40) on the operator that we obtain by quantizing the observable $H^{\prime}=\bar{S} S^{-1} H$ with respect to $\bar{g}$. This yields, that the operator (38) attains in the space in $L^{2}\left(Q, \omega_{\bar{g}}\right)$ the form

$$
U \hat{H} U^{-1}=\left(\bar{S}^{-1} H^{\prime}\right)_{W}(\hat{\bar{x}}, \hat{\bar{p}})=\left(\bar{S}^{-1} \bar{S} S^{-1} H\right)_{W}(\hat{\bar{x}}, \hat{\bar{p}})=\left(S^{-1} H\right)_{W}(\hat{\bar{x}}, \hat{\bar{p}})
$$

Let us thus explicitly calculate the operator on the right hand side of (43). Due to (36) and using the fact that $H$ is second order in momenta (so that the only terms in $S^{-1}$ that act on $H$ are or order up to $\hbar^{2}$, see Remark 4), after some calculations we obtain

$$
S^{-1} H=H+\frac{1}{2} \hbar^{2}\left(\frac{1}{4} A^{i j}{ }_{i j}+\frac{1}{2} A^{i j}{ }_{, i} \Gamma_{j k}^{k}+\frac{1}{2} A^{i j} \Gamma_{i k, j}^{k}+\frac{1}{4} A^{i j} \Gamma_{i k}^{k} \Gamma_{j l}^{l}\right)=\bar{S}^{-1} H+\hbar^{2} W(x)
$$

with

$$
W(x)=\frac{1}{2}\left[\frac{1}{2} A_{, i}^{i j}\left(\Gamma_{j k}^{k}-\bar{\Gamma}_{j k}^{k}\right)+\frac{1}{2} A^{i j}\left(\Gamma_{i k, j}^{k}-\bar{\Gamma}_{i k, j}^{k}\right)+\frac{1}{4} A^{i j}\left(\Gamma_{i k}^{k} \Gamma_{j l}^{l}-\bar{\Gamma}_{i k}^{k} \bar{\Gamma}_{j l}^{l}\right)\right]
$$

coinciding with $W(x)$ in 42).

In Appendix we show that (42) can be written in a covariant form as 


$$
W(x)=\frac{1}{8}\left(A_{; i}^{i j} G^{k s} g_{k s ; j}+A^{i j} G^{k s} g_{k s ; i j}+A^{i j} G_{; i}^{k s} g_{k s ; j}+\frac{1}{4} A^{i j} G^{k r} g_{k r ; i} G^{s l} g_{s l ; j}\right)
$$

where the covariant derivatives are taken with respect to the connection $\bar{\nabla}_{i}$. In what follows we will also need a specification of this correction term to the following situation: suppose that $G=\frac{1}{u} G_{B, \theta}$ (where $u=u(x)$ ) where the metric $G_{B, \theta}$ is flat and suppose that $\bar{G}=G_{B, \theta}$. Then the correction term (45) attains the form

$$
W(x)=\frac{n}{8}\left(A^{i j} \frac{u, j}{u}\right)_{, i}+\frac{n^{2}}{32} \frac{1}{u^{2}} A^{i j} u_{, i} u, j
$$

\section{Separable minimal quantizations of Stäckel systems}

Suppose we have a Stäckel system written in arbitrary Darboux coordinates $(x, p)$ :

$$
H_{r}=\frac{1}{2} p^{T} A_{r} p+V_{r}(x), \quad r=1, \ldots, n
$$

Given a metric $g$ we can now perform the minimal quantization of our Stäckel system (47) as described in the previous section. As a result we obtain $n$ quantum Hamiltonians

$$
\hat{H}_{r}=-\frac{1}{2} \hbar^{2} \nabla_{i}\left(T_{r} G\right)^{i j} \nabla_{j}+V_{r}(x), \quad r=1, \ldots, n
$$

acting in the Hilbert space $L^{2}\left(Q, \omega_{g}\right), \omega_{g}=|\operatorname{det} g|^{1 / 2} d x$, where $A_{r}=T_{r} G$. Let us rewrite the operators (48) in some separation coordinates $(\lambda, \mu)$ for the classical Stäckel system (477). We will always assume the conditions 1-3 from Section 2. This also means that $g$ and thus $G$ are diagonal in separation coordinates. Thus, since $A_{r}$ are diagonal in separation coordinates, so are $T_{r}$. Calculating covariant derivatives we obtain

$$
\begin{aligned}
\hat{H}_{r} & =-\frac{1}{2} \hbar^{2} G^{i i}\left(T_{r}^{(i)} \partial_{i}^{2}+\left(\partial_{i} T_{r}^{(i)}\right) \partial_{i}-T_{r}^{(i)} \Gamma_{i} \partial_{i}\right)+V_{r}(\lambda) \\
& =-\frac{1}{2} \hbar^{2} A_{r}^{i i}\left(\partial_{i}^{2}+\left(\frac{\partial_{i} T_{r}^{(i)}}{T_{r}^{(i)}}-\Gamma_{i}\right) \partial_{i}\right)+V_{r}(\lambda)
\end{aligned}
$$

where $T_{r}^{(i)} \equiv\left(T_{r}\right)_{i}^{i}$ (no summation) and where $\Gamma_{i}$ are metrically contracted Christoffel symbols (9). In orthogonal coordinates they read [4]

$$
\Gamma_{i}=\frac{1}{2} \frac{\partial_{i} \operatorname{det} G}{\operatorname{det} G}-\frac{\partial_{i} G^{i i}}{G^{i i}}
$$

The next theorem, proved in [3], follows directly from (49).

Theorem 7 The necessary and sufficient condition for quantum separability of operators $\hat{H}_{r}$ takes the form

$$
\Xi_{i}=\Xi_{i}\left(\lambda_{i}\right) \text { or } \partial_{j} \Xi_{i}=0, \quad j \neq i
$$

where

$$
\Xi_{i}=\frac{\partial_{i} T_{r}^{(i)}}{T_{r}^{(i)}}-\Gamma_{i}
$$

We will call the condition (50) the generalized Robertson condition. Indeed, due to (20), the operators (49) can then be written as

$$
\hat{H}_{r}=-\frac{1}{2} \hbar^{2}\left(S_{\gamma}^{-1}\right)_{r}^{i} f_{i}\left(\lambda_{i}\right)\left(\partial_{i}^{2}+\Xi_{i}\left(\lambda_{i}\right) \partial_{i}\right)+\left(S_{\gamma}^{-1}\right)_{r}^{i} \sigma_{i}\left(\lambda_{i}\right), \quad r=1, \ldots, n
$$


and then application of the Stäckel matrix $S_{\gamma}$ to the system of eigenvalue problems for (51)

$$
S_{\gamma}\left(\begin{array}{c}
\hat{H}_{1} \Psi \\
\vdots \\
\hat{H}_{n} \Psi
\end{array}\right)=S_{\gamma}\left(\begin{array}{c}
E_{1} \Psi \\
\vdots \\
E_{n} \Psi
\end{array}\right)
$$

separates (52) to $n$ one-dimensional eigenvalue problems

$\left(E_{1} \lambda_{i}^{\gamma_{1}}+E_{2} \lambda_{i}^{\gamma_{2}}+\cdots+E_{n}\right) \psi_{i}\left(\lambda_{i}\right)=-\frac{1}{2} \hbar^{2} f_{i}\left(\lambda_{i}\right)\left[\frac{d^{2} \psi_{i}\left(\lambda_{i}\right)}{d \lambda_{i}^{2}}+\Xi_{i}\left(\lambda_{i}\right) \frac{d \psi_{i}\left(\lambda_{i}\right)}{d \lambda_{i}}\right]+\sigma_{i}\left(\lambda_{i}\right) \psi_{i}\left(\lambda_{i}\right), \quad i=1, \ldots, n$

called separation equations or quantum separable relations, so that

$$
\Psi\left(\lambda_{1}, \ldots, \lambda_{n}, c, E\right)=\prod_{i=1}^{n} \psi_{i}\left(\lambda_{i}, c_{2 i-1,} c_{2 i}, E\right)
$$

is a common, multiplicatively separable solution of stationary Schrödinger equations for all $\hat{H}_{r}$, satisfying the definition of separability from [8]. The constants $E_{i}$ are unspecified unless some boundary conditions are imposed while $c_{2 i-1,}, c_{2 i}$ are integration constants originating during the process of solving equation $i$ in (53); there are $2 n$ of them in total. In the case $G=A_{1}$ (or, in general, $G$ equal to any $A_{s}$ ) $T_{r}$ are Killing tensors of $g$ so in $\lambda$-coordinates $\partial_{i} T_{r}^{(i)}=0$. In consequence the condition (50) reduces to the Robertson condition for quantum separability (7) or (10).

In [18] we proved that for the case $G=A_{1}$ the only class of Stäckel systems (17) for which the Robertson condition (10) is satisfied is the Benenti class where

$$
\Gamma_{i}=-\frac{1}{2} \frac{f_{i}^{\prime}\left(\lambda_{i}\right)}{f_{i}\left(\lambda_{i}\right)}
$$

For all other choices of $\gamma_{i}$ in (17) this condition fails. In [3] we investigated the more general case when $G$ is not one of the tensors $A_{r}$ in (19) but is a flat metric from the Benenti class (26). We showed that also in this case the only class of Stäckel systems (17) that is quantum separable is again the Benenti class. It means that in order to achieve quantum separability of an arbitrary Stäckel system of the type (17) we have to consider a broader class of admissible metric tensors $g$ used in the quantization procedure.

Consider thus a Stäckel system (17) defined by some fixed choice of $\gamma_{1}>\gamma_{2}>\cdots>\gamma_{n}=0$ and the choice of $f_{i}, \sigma_{i}$. We will now search for the metric $G$ that satisfies the generalized Robertson condition (50) for this Stäckel system. Due to the structure (25) of $A_{r}$ we look for $G$ in the form

$$
G=u^{-1}(\lambda) G_{B, \theta}
$$

where $G_{B, \theta}$ is the Benenti metric given by (26) with $n$ arbitrary functions $\theta_{i}\left(\lambda_{i}\right)$ and where $u$ is some function on $Q$. Albeit this choice is by no means the most general one it will prove to be sufficiently general. The tensors $T_{r}$ become in this case

$$
T_{r}=\frac{u}{\varphi} \chi_{r} G_{B, f} g_{B, \theta}
$$

where $\varphi$ is again given by (27) and where as usual $g_{B, \theta}=G_{B, \theta}^{-1}$. Plugging this into (50) we get

$$
\frac{\partial_{i} T_{r}^{(i)}}{T_{r}^{(i)}}-\Gamma_{i}=\frac{\kappa_{i}^{\prime}\left(\lambda_{i}\right)}{\kappa_{i}\left(\lambda_{i}\right)}, \quad=1, \ldots, n
$$

where $\kappa_{i}$ are arbitrary functions of one variable (the right hand side is just a convenient for us way of writing an arbitrary function of $\lambda_{i}$ ). Since for (55)

$$
\Gamma_{i}=\left(\Gamma_{B, \theta}\right)_{i}+\left(1-\frac{1}{2} n\right) \frac{\partial_{i} u}{u}
$$


with $\left(\Gamma_{B, \theta}\right)_{i}$ being the metrically contracted Christoffel symbols for the metric $G_{B, \theta}$, the formula (566) takes the form

$$
\frac{n}{2} \frac{\partial_{i} u}{u}-\frac{\partial_{i} \varphi}{\varphi}=\frac{\kappa_{i}^{\prime}\left(\lambda_{i}\right)}{\kappa_{i}\left(\lambda_{i}\right)}+\frac{1}{2} \frac{\theta_{i}^{\prime}\left(\lambda_{i}\right)}{\theta_{i}\left(\lambda_{i}\right)}-\frac{f_{i}^{\prime}\left(\lambda_{i}\right)}{f_{i}\left(\lambda_{i}\right)}, \quad=1, \ldots, n
$$

which has a solution

$$
u=\varphi^{\frac{2}{n}} \prod_{i=1}^{n}\left(\frac{\left|\theta_{i}\right| \kappa_{i}^{2}}{f_{i}^{2}}\right)^{\frac{1}{n}}
$$

In order to receive a solution as simple as possible we choose $\kappa_{i}$ so that

$$
\frac{\left|\theta_{i}\right| \kappa_{i}^{2}}{f_{i}^{2}}=1
$$

(notice that $\theta_{i}$ are still arbitrary) yielding (57) in the form $u=\varphi^{\frac{2}{n}}$. Thus, we have proved

Theorem 8 Suppose $\theta_{i}, i=1, \ldots, n$ are $n$ arbitrary functions of one variable. Then applying the procedure of minimal quantization, with the metric tensor

$$
g=\varphi^{\frac{2}{n}} g_{B, \theta}
$$

where $g_{B, \theta}=G_{B, \theta}^{-1}$ with $G_{B, \theta}$ given by

$$
G_{B, \theta}=\operatorname{diag}\left(\frac{\theta_{1}\left(\lambda_{1}\right)}{\Delta_{1}}, \ldots, \frac{\theta_{n}\left(\lambda_{n}\right)}{\Delta_{n}}\right)
$$

to the Stäckel system (17) we obtain a quantum separable system (48) with the separation equations of the form

$\left(E_{1} \lambda_{i}^{\gamma_{1}}+E_{2} \lambda_{i}^{\gamma_{2}}+\cdots+E_{n}\right) \psi_{i}\left(\lambda_{i}\right)=-\frac{1}{2} \hbar^{2} f_{i}\left(\lambda_{i}\right)\left[\frac{d^{2} \psi_{i}\left(\lambda_{i}\right)}{d \lambda_{i}^{2}}+\left(\frac{f_{i}^{\prime}\left(\lambda_{i}\right)}{f_{i}\left(\lambda_{i}\right)}-\frac{1}{2} \frac{\theta_{i}^{\prime}\left(\lambda_{i}\right)}{\theta_{i}\left(\lambda_{i}\right)}\right) \frac{d \psi_{i}\left(\lambda_{i}\right)}{d \lambda_{i}}\right]+\sigma_{i}\left(\lambda_{i}\right) \psi_{i}\left(\lambda_{i}\right)$,

where $i=1, \ldots, n$.

The metric $g$ in (58) is a conformal deformation of the Benenti metric $g_{B, \theta}$. Thus, there exists an infinite family of separable quantizations of a Stäckel system (17) parametrized by $n$ arbitrary functions $\theta_{i}$ of one variable: any Stäckel system (17) can be separably quantized in the conformally deformed metric (58) (note that this metric is conformally flat in the case when $g_{B, \theta}$ is flat). Moreover, since for the Benenti class $\varphi=1$, any Stäckel system from the Benenti class (21) can be separably quantized in any metric of Benenti class (59), including the subclass of flat metrics.

\section{Quantum integrability of Stäckel systems in arbitrary Hilbert spaces}

We remind the reader that in [4] the authors derived the necessary and sufficient condition for commutativity of quantum Hamiltonians $\hat{H}_{r}$ of the form (11) (and with $A_{1}=G$ ) called the pre-Robertson condition (12) or (13), which took the form

$$
\partial_{i}^{2} \Gamma_{j}-\Gamma_{i} \partial_{i} \Gamma_{j}=0, \quad i \neq j .
$$

In our case, when $G$ is not related with any $A_{r}$, analogous calculations lead to the following necessary and sufficient condition for commutativity of $\hat{H}_{r}$ which we call the generalized pre-Robertson condition [3]:

$$
\partial_{i}^{2} \Xi_{j}-\Xi_{i} \partial_{i} \Xi_{j}=0, \quad i \neq j .
$$

Assume that we have a Stäckel system $H_{r}, r=1, \ldots, n$ of the form (17). Let us perform the procedure of minimal quantization of this system in the metric $G$ given by (58), as described in the previous section. 
We obtain then the quantum separable system consisting of $n$ operators $\hat{H}_{r}$ acting on the Hilbert space $L^{2}\left(Q, \omega_{g}\right), \omega_{g}=|\operatorname{det} g|^{1 / 2} d \lambda$. Since the generalized Robertson condition (50) implies the generalized pre-Robertson condition (62) we conclude that this system is also quantum integrable: $\left[\hat{H}_{r}, \hat{H}_{s}\right]=0$. Using Theorem 6 we are able to write operators $\hat{H}_{r}$ in another metric $\bar{g}$ i.e. in the Hilbert space $L^{2}\left(Q, \omega_{\bar{g}}\right)$ which yields new quantum operators $\hat{\bar{H}}_{r}, r=1, \ldots, n$ that constitute again quantum integrable (but not necessarily quantum separable) system. Due to the theory developed in Section 5 we know, that we can equally well take the classical Hamiltonians $H_{r}$ amended by quantum correction terms, i.e. the functions $H_{r}+\hbar^{2} W_{r}$ with $W_{r}$ given by (42) (or equivalently by (45)) and minimally quantize them in the metric $\bar{g}$ as this will yield the same quantum integrable system $\hat{\bar{H}}_{r}, r=1, \ldots, n$.

In [19] we demonstrated that any Stäckel system of the class (17) can be constructed by an appropriate Stäckel transform of a suitably chosen flat Stäckel system from Benenti class. Moreover, in 20] we explicitly constructed flat coordinates for any flat Stäckel system. Therefore we are able to write down our original Stäckel system $H_{r}, r=1, \ldots, n$ in flat coordinates of the metric $\bar{g}$ of the form (26) ( $\bar{g}$ is flat as soon the conditions in Remark 3 are satisfied). In this specific case, if we apply the standard Weyl quantization to the Stäckel system $H_{r}+\hbar^{2} W_{r}$ (i.e. our original system amended by the quantum correction terms $\hbar^{2} W_{r}$ ) we will obtain a quantum integrable system. One can also say, alternatively, that if we want to avoid quantum correction terms, we should quantize the original system $H_{r}, r=1, \ldots, n$ not by Weyl quantization but by minimal quantization in a suitably chosen conformally flat metric $G$.

In papers [1] and 2] the authors presented some ad hoc calculations generating quantum correction terms that guarantee integrability of quantum systems obtained through Weyl quantization of some Hamiltonian systems. Our theory shows how to construct these quantum correction terms in a systematic way (albeit within the class of Stäckel systems, not considered in [1]-[2]). We will illustrate this on two examples below. It is important to stress that the presented systems cannot be separably quantized in the frame of the classical Robertson-Eisenhart formalism.

Example 9 Consider the Stäckel system (17) for $n=3$ given by the separation relations of the form:

$$
H_{1} \lambda_{i}^{3}+H_{2} \lambda_{i}+H_{3}=\frac{1}{2} \lambda_{i} \mu_{i}^{2}+\lambda_{i}^{4}, \quad i=1,2,3
$$

so that $\gamma_{1}=3, \gamma_{2}=1$ and $\gamma_{3}=0$ and with $f_{i}\left(\lambda_{i}\right)=\lambda_{i}$ and $\sigma_{i}\left(\lambda_{i}\right)=\lambda_{i}^{4}$. In this case $\varphi=\rho_{1}(\lambda)=$ $-\left(\lambda_{1}+\lambda_{2}+\lambda_{3}\right)$. Consider also the corresponding metric $G_{B, f}$ given by (26). This metric is flat, by Remark 3 . In the coordinates $x_{1}, x_{2}, x_{3}$ defined through (cf. 24))

$$
\begin{gathered}
\rho_{1} \equiv-\left(\lambda_{1}+\lambda_{2}+\lambda_{3}\right)=x_{1} \\
\rho_{2} \equiv \lambda_{1} \lambda_{2}+\lambda_{1} \lambda_{3}+\lambda_{2} \lambda_{3}=x_{2}+\frac{1}{4} x_{1}^{2} \\
\rho_{3} \equiv-\lambda_{1} \lambda_{2} \lambda_{3}=-\frac{1}{4} x_{3}^{2}
\end{gathered}
$$

the metric $G_{B, f}$ reads

$$
G_{B, f}=\left(\begin{array}{ccc}
0 & 1 & 0 \\
1 & 0 & 0 \\
0 & 0 & 1
\end{array}\right)
$$

so $\varphi=x_{1}$ in $x_{i}$-coordinates and $x_{i}$ are flat non-orthogonal coordinates for $G_{B, f}$. Solving the relations (63) with respect to the Hamiltonians $H_{i}$ and passing to the variables $x_{i}$ we receive $H_{r}=A_{r}^{i j} y_{i} y_{j}+V_{r}(x)$ where $y_{i}$ are momenta conjugate to $x_{i}$ and where the tensors $A_{r}$ have the form

$$
\begin{gathered}
A_{1}=\left(\begin{array}{ccc}
0 & -\frac{1}{x_{1}} & 0 \\
-\frac{1}{x_{1}} & 0 & 0 \\
0 & 0 & -\frac{1}{x_{1}}
\end{array}\right), \quad A_{2}=\left(\begin{array}{ccc}
1 & \frac{1}{4} x_{1}-\frac{x_{2}}{x_{1}} & 0 \\
\frac{1}{4} x_{1}-\frac{x_{2}}{x_{1}} & -x_{2} & -\frac{1}{2} x_{3} \\
0 & -\frac{1}{2} x_{3} & \frac{3}{4} x_{1}-\frac{x_{2}}{x_{1}}
\end{array}\right), \\
A_{3}=\left(\begin{array}{ccc}
0 & \frac{1}{4} \frac{x_{3}^{2}}{x_{1}} & -\frac{1}{2} x_{3} \\
\frac{1}{4} \frac{x_{3}^{2}}{x_{1}} & \frac{1}{4} x_{3}^{2} & -\frac{1}{4} x_{1} x_{3} \\
-\frac{1}{2} x_{3} & -\frac{1}{4} x_{1} x_{3} & \frac{1}{4} x_{1}^{2}+x_{2}+\frac{1}{4} \frac{x_{3}^{2}}{x_{1}}
\end{array}\right)
\end{gathered}
$$


with the corresponding rational potentials

$$
\begin{aligned}
& V_{1}(x)=-\frac{3}{4} x_{1}+\frac{x_{2}}{x_{1}} \\
& V_{2}(x)=\frac{1}{16} x_{1}^{3}+\frac{1}{2} x_{1} x_{2}+\frac{1}{4} x_{3}^{2}+\frac{x_{2}^{2}}{x_{1}} \\
& V_{3}(x)=-\frac{1}{16} x_{1} x_{3}^{2}-\frac{1}{4} \frac{x_{2} x_{3}^{2}}{x_{1}}
\end{aligned}
$$

From our theory it follows that we can perform a separable quantization of this system in the conformally flat metric $G=\frac{1}{u} G_{B, f}$ (which means that we choose $\theta_{i}=f_{i}$ ) with $u=\varphi^{2 / n}=x_{1}^{2 / 3}$. We obtain three commuting operators

$$
\hat{H}_{r}=-\frac{1}{2} \hbar^{2} \nabla_{i} A_{r}^{i j} \nabla_{j}+V_{r}(x)
$$

(where $\nabla_{i}$ is the connection defined by $\left.G\right)$, acting in the Hilbert space $L^{2}\left(Q, \omega_{g}\right)=L^{2}\left(Q,\left|x_{1}\right| d x\right)\left(\omega_{g}=\right.$ $\left.|\operatorname{det} g|^{1 / 2} d x=\left|u^{3 / 2}\right| d x=\left|x_{1}\right| d x\right)$. In the separation coordinates $(\lambda, \mu)$ the separation equations (60) for $\hat{H}_{r}$ attain the form

$$
\left(E_{1} \lambda_{i}^{3}+E_{2} \lambda_{i}+E_{3}\right) \psi_{i}\left(\lambda_{i}\right)=-\frac{1}{2} \hbar^{2}\left(\lambda_{i} \frac{d^{2} \psi_{i}\left(\lambda_{i}\right)}{d \lambda_{i}^{2}}+\frac{1}{2} \frac{d \psi_{i}\left(\lambda_{i}\right)}{d \lambda_{i}}\right)+\lambda_{i}^{4} \psi_{i}\left(\lambda_{i}\right), \quad i=1,2,3
$$

Let us now rewrite our operators ([66) in the Hilbert space $L^{2}\left(Q, \omega_{\bar{g}}\right)=L^{2}(Q, d x)\left(\omega_{\bar{g}}=|\operatorname{det} \bar{g}|^{1 / 2} d x=d x\right)$ with the flat metric $\bar{G}=G_{B, f}$. From our theory it follows that a suitable way to do it is to quantize our Hamiltonians $H_{r}$ directly in the metric $\bar{G}$ after amending them by the quantum correction terms $W_{i}(x)$ given by (46)

$$
W_{1}=0, W_{2}=-\frac{3}{8} \frac{1}{x_{1}^{2}}, W_{3}=-\frac{1}{8} \frac{1}{x_{1}}
$$

One can check by direct calculations that the operators

$$
\hat{\bar{H}}_{r}=-\frac{1}{2} \hbar^{2} \partial_{i} A_{r}^{i j} \partial_{j}+\hbar^{2} W_{r}(x)+V_{r}(x), \quad r=1, \ldots, n
$$

(the coordinates $x_{i}$ are flat for $\bar{g}=G_{B, f}$ so $\bar{\nabla}_{i}=\partial_{i}=\partial / \partial x_{i}$ ) do indeed commute, thus constituting a quantum integrable system. The operators [68) are however not quantum separable, contrary to the operators (66), but are $R$-separable. It means that in separation coordinates

$$
\widehat{\bar{H}}_{r} \bar{\Psi}(\lambda)=E_{r} \bar{\Psi}(\lambda), \quad \bar{\Psi}(\lambda)=U(\lambda) \Psi(\lambda)=\left(\lambda^{1}+\lambda^{2}+\lambda^{3}\right)^{\frac{1}{2}} \psi\left(\lambda^{1}\right) \psi\left(\lambda^{2}\right) \psi\left(\lambda^{3}\right),
$$

and $\psi\left(\lambda^{i}\right)$ solves 67$)$.

Example 10 In our second example we consider the following Stäckel system

$$
H_{1} \lambda_{i}^{3}+H_{2} \lambda_{i}^{2}+H_{3}=\frac{1}{2} \lambda_{i} \mu_{i}^{2}+\lambda_{i}^{4}, \quad i=1,2,3
$$

so that this time $\gamma_{1}=3, \gamma_{2}=2$ and $\gamma_{3}=0$ but still with $f_{i}\left(\lambda_{i}\right)=\lambda_{i}$ and $\sigma_{i}\left(\lambda_{i}\right)=\lambda_{i}^{4}$. In this case $\varphi=\rho_{2}(\lambda)=\lambda_{1} \lambda_{2}+\lambda_{1} \lambda_{3}+\lambda_{2} \lambda_{3}$. We consider again the same metric $G_{B, f}$ with the same flat coordinates $x_{i}$ given by 64). This time the tensors $A_{r}$ have the form

$$
\begin{aligned}
& A_{1}=\frac{1}{\rho_{2}(x)}\left(\begin{array}{ccc}
-1 & -\frac{1}{2} x_{1} & 0 \\
-\frac{1}{2} x_{1} & x_{2} & \frac{1}{2} x_{3} \\
0 & \frac{1}{2} x_{3} & -x_{1}
\end{array}\right), \quad A_{2}=\frac{1}{\rho_{2}(x)}\left(\begin{array}{ccc}
-x_{1} & -\frac{1}{4} x_{1}^{2}+x_{2} & 0 \\
-\frac{1}{4} x_{1}^{2}+x_{2} & x_{2} x_{1} & \frac{1}{2} x_{1} x_{3} \\
0 & \frac{1}{2} x_{1} x_{3} & -\frac{3}{4} x_{1}^{2}+x_{2}
\end{array}\right) \\
& A_{3}=\frac{1}{4 \rho_{2}(x)}\left(\begin{array}{ccc}
x_{3}{ }^{2} & \frac{1}{2} x_{3}{ }^{2} x_{1} & -\frac{1}{2}\left(x_{1}{ }^{2}+4 x_{2}\right) x_{3} \\
\frac{1}{2} x_{3}{ }^{2} x_{1} & \frac{1}{4} x_{3}{ }^{2} x_{1}{ }^{2} & -\frac{1}{4} x_{3}\left(x_{1}{ }^{3}+4 x_{2} x_{1}+2 x_{3}{ }^{2}\right) \\
-\frac{1}{2}\left(x_{1}{ }^{2}+4 x_{2}\right) x_{3} & -\frac{1}{4} x_{3}\left(x_{1}{ }^{3}+4 x_{2} x_{1}+2 x_{3}{ }^{2}\right) & \frac{1}{4} x_{1}{ }^{4}+2 x_{1}{ }^{2} x_{2}+4 x_{2}{ }^{2}+x_{3}{ }^{2} x_{1}
\end{array}\right)
\end{aligned}
$$


where $\rho_{2}(x)=x_{2}+\frac{1}{4} x_{1}^{2}$, while the potentials are

$$
\begin{aligned}
& V_{1}(x)=-\frac{1}{4 \rho_{2}(x)}\left(x_{1}^{3}+4 x_{1} x_{2}+x_{3}^{2}\right) \\
& V_{2}(x)=-\frac{1}{4 \rho_{2}(x)}\left(\frac{1}{4} x_{1}^{4}+2 x_{1}^{2} x_{2}+x_{1} x_{3}^{2}+4 x_{2}^{2}\right) \\
& V_{3}(x)=\frac{x_{3}^{4}}{16 \rho_{2}(x)}
\end{aligned}
$$

This time we perform a separable quantization in the conformally flat metric $G=\frac{1}{u} G_{B, f}$ with $u=\varphi^{2 / n}=$ $\left(x_{2}+\frac{1}{4} x_{1}^{2}\right)^{2 / 3}$. We obtain again three commuting operators

$$
\hat{H}_{r}=-\frac{1}{2} \hbar^{2} \nabla_{i} A_{r}^{i j} \nabla_{j}+V_{r}(x)
$$

(where $\nabla_{i}$ is the connection defined by $G$ ), acting in the Hilbert space $L^{2}\left(Q,\left|\rho_{2}(x)\right| d x\right)$, while the separation equations (60) for $\hat{H}_{r}$ become

$$
\left(E_{1} \lambda_{i}^{3}+E_{2} \lambda_{i}^{2}+E_{3}\right) \psi_{i}\left(\lambda_{i}\right)=-\frac{1}{2} \hbar^{2}\left(\lambda_{i} \frac{d^{2} \psi_{i}\left(\lambda_{i}\right)}{d \lambda_{i}^{2}}+\frac{1}{2} \frac{d \psi_{i}\left(\lambda_{i}\right)}{d \lambda_{i}}\right)+\lambda_{i}^{4} \psi_{i}\left(\lambda_{i}\right), \quad i=1,2,3
$$

with the same right hand side as in the previous example. Rewriting our operators (66) in the Hilbert space $L^{2}(Q, d x)$ with quantization defined by the flat metric $\bar{G}=G_{B, f}$ leads to the following correction terms $W_{i}(x)$

$$
\begin{aligned}
& W_{1}=\frac{1}{16 \rho_{2}^{3}(x)}\left(5 x_{1}^{2}-4 x_{2}\right) \\
& W_{2}=\frac{1}{32 \rho_{2}^{3}(x)}\left(7 x_{1}^{3}-20 x_{1} x_{2}\right) \\
& W_{3}=-\frac{1}{128 \rho_{2}^{3}(x)}\left(x_{1}^{5}+8 x_{1}^{3} x_{2}+13 x_{1}^{2} x_{3}^{2}+16 x_{1} x_{2}^{2}+4 x_{2} x_{3}^{2}\right)
\end{aligned}
$$

Again, the operators

$$
\hat{\bar{H}}_{r}=-\frac{1}{2} \hbar^{2} \partial_{i} A_{r}^{i j} \partial_{j}+\hbar^{2} W_{r}(x)+V_{r}(x), \quad r=1, \ldots, n
$$

commute, as it can be checked for example in Maple. Operators (72) are R-separable and in separation coordinates

$$
\widehat{\bar{H}}_{r} \bar{\Psi}(\lambda)=E_{r} \bar{\Psi}(\lambda), \quad \bar{\Psi}(\lambda)=U(\lambda) \Psi(\lambda)=\left(\lambda^{1} \lambda^{2}+\lambda^{1} \lambda^{3}+\lambda^{2} \lambda^{3}\right)^{\frac{1}{2}} \psi\left(\lambda^{1}\right) \psi\left(\lambda^{2}\right) \psi\left(\lambda^{3}\right),
$$

where $\psi\left(\lambda^{i}\right)$ solves [71).

\section{Appendix}

We sketch here the proof of the fact that formulas (42) and (45) are equivalent. We want to demonstrate that

$$
W(x)=\frac{1}{8}\left[A^{i j}\left(\Gamma_{i k}^{k} \Gamma_{j s}^{s}-\bar{\Gamma}_{i k}^{k} \bar{\Gamma}_{j s}^{s}\right)+2\left(A^{i j}\left(\Gamma_{j k}^{k}-\bar{\Gamma}_{j k}^{k}\right)\right)_{, i}\right]
$$

or, equivalently

$$
W(x)=\frac{1}{2}\left[\frac{1}{2} A^{i j}{ }_{, i}\left(\Gamma_{j k}^{k}-\bar{\Gamma}_{j k}^{k}\right)+\frac{1}{2} A^{i j}\left(\Gamma_{i k, j}^{k}-\bar{\Gamma}_{i k, j}^{k}\right)+\frac{1}{4} A^{i j}\left(\Gamma_{i k}^{k} \Gamma_{j l}^{l}-\bar{\Gamma}_{i k}^{k} \bar{\Gamma}_{j l}^{l}\right)\right]
$$

coincides with 


$$
W(x)=\frac{1}{8}\left(A_{; i}^{i j} G^{k s} g_{k s ; j}+A^{i j} G^{k s} g_{k s ; i j}+A^{i j} G_{; i}^{k s} g_{k s ; j}+\frac{1}{4} A^{i j} G^{k r} g_{k r ; i} G^{s l} g_{s l ; j}\right)
$$

where the covariant differentiation is taken with respect to the metric $\bar{g}$. To this end, denote by $M_{j}^{i}$ the quotient of $\bar{g}_{i j}$ and $g_{i j}$ :

$$
\bar{g}_{i j}=M_{i}^{k} g_{k j},
$$

(as such, it is a $(1,1)$-tensor), which yields

$$
G^{i j}=\left(M^{-1}\right)_{k}^{i} \bar{g}^{k j}
$$

The Christoffel symbols $\Gamma_{j k}^{i}$ can now be expressed through $\bar{\Gamma}_{j k}^{i}$ in the following way

$$
\begin{aligned}
\Gamma_{j k}^{i} & =\frac{1}{2} G^{i l}\left(g_{l j, k}+g_{l k, j}-g_{j k, l}\right) \\
& =\frac{1}{2}\left(M^{-1}\right)_{r}^{i} \bar{g}^{r l}\left(M_{l, k}^{s} \bar{g}_{s j}+M_{l}^{s} \bar{g}_{s j, k}+M_{l, j}^{s} \bar{g}_{s k}+M_{l}^{s} \bar{g}_{s k, j}-M_{j, l}^{s} \bar{g}_{s k}-M_{j}^{s} \bar{g}_{s k, l}\right)
\end{aligned}
$$

yielding

$$
\begin{aligned}
\Gamma_{j k}^{i}= & \bar{\Gamma}_{j k}^{i}+\frac{1}{2}\left(M^{-1}\right)_{r}^{i} M_{l, k}^{s} \bar{g}^{r l} \bar{g}_{s j}+\frac{1}{2}\left(M^{-1}\right)_{r}^{i} M_{l, j}^{s} \bar{g}^{r l} \bar{g}_{s k}-\frac{1}{2}\left(M^{-1}\right)_{r}^{i} M_{j, l}^{s} \bar{g}^{r l} \bar{g}_{s k} \\
& -\frac{1}{2}\left(M^{-1}\right)_{r}^{i} M_{j}^{s} \bar{g}^{r l} \bar{g}_{s k, l}+\frac{1}{2} \bar{g}^{i s} \bar{g}_{j k, s} .
\end{aligned}
$$

Using

$$
\begin{aligned}
0 & =\bar{g}_{j k ; s}=\bar{g}_{j k, s}-\bar{g}_{n k} \bar{\Gamma}_{j s}^{n}-\bar{g}_{j n} \bar{\Gamma}_{k s}^{n}, \\
M_{l, k}^{s} & =M_{l ; k}^{s}-M_{l}^{n} \bar{\Gamma}_{n k}^{s}+M_{n}^{s} \bar{\Gamma}_{l k}^{n}
\end{aligned}
$$

(where ; denotes the covariant differentiation with respect to $\bar{g}$ ) we receive

$$
\Gamma_{j k}^{i}=\bar{\Gamma}_{j k}^{i}+\frac{1}{2}\left(M^{-1}\right)_{r}^{i} M_{j ; k}^{r}+\frac{1}{2}\left(M^{-1}\right)_{r}^{i} M_{k ; j}^{r}-\frac{1}{2}\left(M^{-1}\right)_{r}^{i} M_{j ; l}^{s} \bar{g}^{r l} \bar{g}_{s k} .
$$

In particular

$$
\Gamma_{j k}^{k}=\bar{\Gamma}_{j k}^{k}+\frac{1}{2}\left(M^{-1}\right)_{r}^{k} M_{k ; j}^{r}
$$

Moreover

$$
A_{, i}^{i j}=A_{; i}^{i j}-\bar{\Gamma}_{s i}^{j} A^{i s}-\bar{\Gamma}_{s i}^{i} A^{s j}
$$

Inserting all this into (74) we obtain

$$
W=\frac{1}{8}\left(A_{; i}^{i j}\left(M^{-1}\right)_{r}^{k} M_{k ; j}^{r}+A^{i j}\left(M^{-1}\right)_{r}^{k} M_{k ; i j}^{r}+A^{i j}\left(M^{-1}\right)_{r ; i}^{k} M_{k ; j}^{r}+\frac{1}{4} A^{i j}\left(M^{-1}\right)_{r}^{k} M_{k ; i}^{r}\left(M^{-1}\right)_{s}^{l} M_{l ; j}^{s}\right)
$$

that due to (76) coincides with (75).

\section{Acknowledgments}

Z. Domański acknowledge the support of Polish National Science Center grant under the contract number DEC-2011/02/A/ST1/00208. 


\section{References}

[1] Hietarinta, J. Classical versus quantum integrability. J. Math. Phys. 25 (1984), no. 6, 1833-1840.

[2] Hietarinta, J.; Grammaticos, B. On the $\hbar^{2}$ correction terms in quantum integrability. J. Phys. A 22 (1989), no. 9, 1315-1322.

[3] Błaszak, M.; Domański, Z,; Silindir, B. Flat minimal quantizations of Stäckel systems and quantum separability. Ann. Physics 351 (2014), 152-165.

[4] Benenti, S.; Chanu, C.; Rastelli, G. Remarks on the connection between the additive separation of the Hamilton-Jacobi equation and the multiplicative separation of the Schrödinger equation. II. First integrals and symmetry operators. J. Math. Phys. 43 (2002), no. 11, 5223-5253.

[5] Stäckel, P. Die Integration der Hamilton-Jacobischen Differentialgleichung mittelst Separation der Variablen. Habilitationsschrift, Halle 1891.

[6] Robertson, H. P. Bemerkung über separierbare Systeme in der Wellenmechanik. Math. Ann. 98 (1928), no. 1, 749-752.

[7] Eisenhart, L. P. Separable systems of Stäckel. Ann. of Math. 35 (1934), no. 2, 284-305.

[8] Benenti, S.; Chanu, C.; Rastelli, G. Remarks on the connection between the additive separation of the Hamilton-Jacobi equation and the multiplicative separation of the Schrödinger equation. I. The completeness and Robertson conditions. J. Math. Phys. 43 (2002), no. 11, 5183-5222.

[9] Błaszak, M.; Domański, Z. Phase space quantum mechanics. Ann. Phys. 327 (2012), no. 2, 167-211.

[10] Błaszak, M.; Domański, Z. Canonical quantization of classical mechanics in curvilinear coordinates. Invariant quantization procedure. Ann. Phys. 339 (2013), 89-108.

[11] Błaszak, M.; Domański, Z. Natural star-products on symplectic manifolds and related quantum mechanical operators. Ann. Phys. 344 (2014), 29-42.

[12] Sklyanin, E. K. Separation of variables - new trends. Progr. Theoret. Phys. Suppl. 118 (1995), $35-60$.

[13] Błaszak, M. Separable systems with quadratic in momenta first integrals. J. Phys. A 38 (2005), no. 8, 1667-1685.

[14] Błaszak, M.; Marciniak, K. Stäckel systems generating coupled KdV hierarchies and their finite-gap and rational solutions. J. Phys. A 41 (2008), no. 48, 485202.

[15] Marciniak, K.; Błaszak, M. Construction of coupled Harry Dym hierarchy and its solutions from Stäckel systems. Nonlinear Analysis: Theory, Methods and Applications 73 (2010) 3004-3017, http://dx.doi.org/10.1016/j.na.2010.06.067.

[16] DeWitt, B. S. Point Transformations in Quantum Mechanics, Phys. Rev. 85, 653 (1952)

[17] Essén, H. Quantization and independent coordinates, Am. J. Phys. 46, 983 (1978)

[18] Błaszak, M.; Domański, Z.; Sergyeyev, A.; Szablikowski, B. M. Integrable quantum Stäckel systems. Phys. Lett. A 377 (2013), no. 38, 2564-2572.

[19] Błaszak, M.; Marciniak, K. On reciprocal equivalence of Stäckel systems. Stud. Appl. Math. 129 (2012), no. 1, 26-50.

[20] Marciniak, K.; Błaszak, M. Flat coordinates for flat Stäckel systems. Applied Mathematics and Computation 268 (2015), 706-716 